\title{
A characteristic ganglioside antibody pattern in the CSF of patients with amyotrophic lateral sclerosis
}

\author{
Andreas Stevens, Michael Weller, Horst Wiethölter
}

\begin{abstract}
Paired cerebrospinal fluid and serum samples of patients with amyotrophic lateral sclerosis $(n=35)$ revealed no consistent abnormalities of CSF cell count, CSF albumin, CSF IgG, CSF IgM, IgG or IgM index, or oligoclonal immunoglobulin band formation in the CSF. Determination of IgG and IgM CSF and serum antibodies to gangliosides GM1, GM2, GM3, AGM1, GD1a, GD1b, and GT1b showed a characteristic pattern which allowed the differentiation of amyotrophic lateral sclerosis from controls and from patients with other neurological disorders including multiple sclerosis. Specifically, patients with the disease had elevated CSF IgM antibodies to all gangliosides except AGM1. The lack of correlation between the CSF findings and corresponding serum antibodies suggests a chronic, compartmental, intrathecal immune response of low activity in amyotrophic lateral sclerosis. Whether this immune response is primary and of pathogenetic signifcance, or an epiphenomenon of neuronal degeneration, remains to be investigated.
\end{abstract}

(F Neurol Neurosurg Psychiatry 1993;56:361-364)

Amyotrophic lateral sclerosis is characterised by a progressive loss of upper and lower motor neurons of unknown aetiology. ${ }^{1}$ Cerebrospinal fluid studies are scarce and have produced no consistent abnormalities in patients with this disease..$^{23}$ Several authors have postulated that a disturbance of immunoregulation may play a role in the degeneration of motor neurons in these cases. ${ }^{4-7}$ The detection of polyclonal serum antibodies to GM1 ganglioside in such patients $^{89}$ has reinforced the search for immunological events in the pathogenesis of the disease. As the pathological changes are largely confined to the central nervous system and because there is no significant bloodbrain barrier disturbance, studies on the CSF are more likely to establish immune dysfunction than investigation of the systemic immune response. This study reports an analysis of CSF and serum IgG and IgM antibodies to seven different gangliosides in 35 patients with amyotrophic lateral sclerosis and examines their correlation with routine CSF parameters.
Materials and methods

Thirty five patients with amyotrophic lateral sclerosis, 16 men and 19 women, mean age $53.5(2.4)$ years, range 25-79 years at diagnosis, without a family history of the illness, were included in this study. The diagnosis was clinical and based on the presence of both upper and lower motor neuron symptoms and signs. Eight patients had prominent bulbar signs, 31 had definite spasticity. For inclusion in this study, the onset of symptoms had to be insidious and not started before the age of 20. The clinical course had to be progressive. Specific attention was given to alternative diagnoses of cervical spondylosis, neoplastic lesions of cervical spinal cord and brain stem, polymyositis, peripheral neuropathy, progressive muscle atrophy, Charcot-Marie-Tooth disease, spinocerebellar degeneration and familial spastic paraplegia. CSF and serum samples were obtained after informed consent, centrifuged and stored at $-70^{\circ} \mathrm{C}$.

CSF parameters and ganglioside antibodies were estimated as previously described. ${ }^{10-13}$ Briefly, micro-enzyme linked immunosorbent assay (ELISA) plates (M129A, Dynatech, Denkendorf, Germany) were coated with purified ganglioside antigen, GM1, GM2, GM3, AGM1, GDla, GD1b, or GT1b (150 ng per well, Sigma, St Louis, MO, United States) diluted in methanol. Antigen purity was confirmed by thin layer chromatography. Protein binding sites on the well that might cause non-specific binding of immunoglobulins were blocked by incubation with bovine serum albumin $(0.1 \%)$ in phosphate-buffered saline for 24 hours before the incubation of CSF or serum samples. The CSF samples were matched to an IgG concentration of $5 \mathrm{mg} / \mathrm{l}$. Serum was diluted 1:50 in phosphate-buffered saline. No detergents were used throughout the assay. IgG or IgM ganglioside reactive antibodies were labelled by anti-human IgG (Sigma A-5403) or antihuman IgM (Sigma A-3914) antisera conjugated to alkaline phosphatase diluted 1:1000 in phosphate-buffered saline. Monoclonality of positive samples was excluded by differential assays for $\kappa$ or $\lambda$ light chains as previously described. ${ }^{12}$

Seven internal reference sera obtained from healthy volunteers or seven CSF samples from patients with suspected, but eventually disproven, neurological disease served as a control for interassay variability. The interassay correlation calculated for nine runs was 0.91 . The ratio of the optical density of a 
Table 1 Mean normalised optical density readings (SD) for ganglioside antibodies in CSF (adjusted to $5 \mathrm{mg} / \mathrm{lg} \mathrm{Ig}$ ) and serum (diluted 1:50) of patients with amyotrophic lateral sclerosis and controls with other neurological diseases.

\begin{tabular}{|c|c|c|c|c|}
\hline$A L S C S F$ & OND CSF & $A L S$ serum & $O N D$ serum & $\begin{array}{l}\text { Controls } \\
\text { serum }\end{array}$ \\
\hline GM1-IgG & $1.09(0.38)$ & $1.00(0.15)$ & $1.08(0.28)$ & $1.00(0.14)$ \\
\hline GM1-IgM & $1.17(0.17)^{\star}$ & $1.06(0.15)$ & $1.09(0.34)$ & $1.00(0.28)$ \\
\hline GM2-IgG & $0.94(0.14)$ & $1.04(0.11)$ & $1.16(0.21)^{\star}$ & $1.00(0.16)$ \\
\hline GM2-IgM & $1.22(0.25)$ & $1.00(0.14)$ & $1 \cdot 12(0 \cdot 36)$ & $1.00(0.28)$ \\
\hline GM3-IgG & $0.91(0.18)$ & $1.00(0.11)$ & $1.11(0.27)^{\star}$ & $1.00(0.19)$ \\
\hline GM3-IgM & $1.30(0.37)^{\star}$ & $1.00(0.11)$ & $1.13(0.33)$ & $1.00(0.25)$ \\
\hline AGM1-IgG & $0.94(0.17)$ & $0.99(0.14)$ & $1.21(0.34)^{\star}$ & $1.00(0.20)$ \\
\hline AGM1-IgM & $1.08(0.16)$ & $0.99(0.06)$ & $1 \cdot 10(0.34)$ & $1.00(0.21)$ \\
\hline GD1a-IgG & $1.05(0.17)$ & $1.01(0.12)$ & $1.06(0.18)$ & $1.00(0.15)$ \\
\hline GD1a-IgM & $1.22(0.28)^{\star}$ & $0.98(0.08)$ & $1.00(0.31)$ & $1.00(0.27)$ \\
\hline GD1b-IgG & $0.95(0.16)$ & $1.00(0.12)$ & $1.06(0.18)^{\star}$ & $1.00(0.13)$ \\
\hline GDlb-IgM & $1.57(0.49)^{\star}$ & $1.00(0.06)$ & $1.13(0.39)$ & $1.00(0.26)$ \\
\hline GT1b-IgG & $1 \cdot 18(0.25)$ & $1.00(0.13)$ & $1.20(0.20)$ & $1.00(0.13)$ \\
\hline GT1b-IgM & $1.47(0.40)^{\star}$ & $1.00(0 \cdot 10)$ & $1.05(0.32)$ & $1.00(0.26)$ \\
\hline
\end{tabular}

ALS = amyotrophic lateral sclerosis; OND = other neurological diseases.

*Significantly $(\mathrm{p}<0.03)$ elevated readings in patients with amyotrophic lateral sclerosis compared with those with other neurological diseases.

Table 2 Incidence of pathological CSF and serum ganglioside antibodies (normalised optical density readings beyond the third percentile) in patients with amyotrophic lateral sclerosis.

\begin{tabular}{|c|c|c|c|c|c|c|c|c|}
\hline & \multicolumn{2}{|c|}{$C S F \operatorname{Ig} G$} & \multicolumn{2}{|c|}{$I g M$} & \multicolumn{2}{|c|}{ Serum IgG } & \multicolumn{2}{|l|}{$I g M$} \\
\hline & $n$ & $\%$ & $n$ & $\%$ & $n$ & $\%$ & $n$ & $\%$ \\
\hline GM1 & 3 & 9 & 5 & 14 & 5 & 14 & 4 & 11 \\
\hline GM2 & 1 & 3 & 10 & 29 & 5 & 14 & 3 & 9 \\
\hline GM3 & 2 & 6 & 21 & 60 & 3 & 9 & 5 & 14 \\
\hline AGM1 & 0 & 0 & 9 & 26 & 5 & 14 & 6 & 17 \\
\hline GDla & 3 & 9 & 13 & 37 & 2 & 6 & 1 & 3 \\
\hline GDib & 1 & 3 & 27 & 77 & 5 & 14 & 5 & 14 \\
\hline GTib & 10 & 29 & 24 & 69 & 13 & 37 & 2 & 6 \\
\hline
\end{tabular}

Figure 1 Individual normalised optical density readings for CSF IgM ganglioside antibodies in patients with (left) amyotrophic lateral sclerosis and (right) multiple sclerosis. patient sample over the mean optical density of the seven internal standards was calculated, thus arbitrarily assigning the value of one to this mean. These normalised optical density results obtained in amyotrophic lateral sclerosis were then compared with a control group of patients with other neurological diseases $(n=24)$ such as tension headache or normal pressure hydrocephalus. Patients with neuroinflammatory and neuroimmunological disorders were excluded. The means of the actual optical density readings in the group with other neurological diseases at $30 \mathrm{~min}$ utes were between 0.072 and 0.079 for the seven CSF IgM ganglioside antibodies, between 0.138 and 0.191 for CSF IgG, between 0.294 and 0.462 for serum IgM, and between 0.265 and 0.394 for serum IgG.

The results in amyotrophic lateral sclerosis were also compared with two groups of previously characterised ${ }^{13}$ patients with multiple

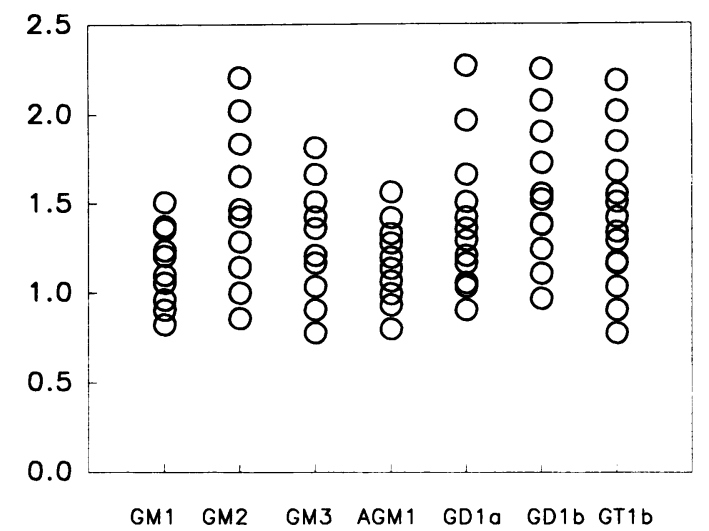

sclerosis of chronic progressive $(n=13)$ and relapsing remitting type $(n=35)$. The statistical analysis included testing for normal distribution, Student's $t$ test, linear correlation, discriminant analysis, and analysis of variance.

\section{Results}

The routine laboratory investigations of CSF and serum samples from amyotrophic lateral sclerosis did not yield consistent abnormalities. None of the patients had a paraprotein on serum immune electrophoresis. Patients with the disease did not have higher total serum IgG or IgM levels than controls. There were also no significant differences between patients and controls in CSF cell counts, CSF albumin, CSF IgG, CSF IgM, or IgG or IgM index. Seven patients had elevated CSF IgG, one had elevated CSF albumin, six had mild pleocytosis. Two had CSF IgG oligoclonal immunoglobulin bands detected. CSF IgG and IgG index in these patients were normal.

The results of CSF and serum ganglioside antibody determination in amyotrophic lateral sclerosis and the incidence of pathological normalised optical density readings in these patients are provided in tables 1 and 2 . The controls with other neurological diseases were not different from the internal controls. CSF IgG, but not age or gender, had an influence on ganglioside antibodies in those with other neurological diseases, before adjustment. The Pearson coefficients were $0 \cdot 14$ (GM1), 0.56 (GM2), 0.37 (GM3), 0.23 (AGM1), 0.48 (GD1a), 0.47 (GDlb) and 0.35 (GT1b). Among the seven ganglioside antibodies examined, there was considerable intercorrelation in the amyotrophic lateral sclerosis samples (Pearson coefficient $0 \cdot 3-0 \cdot 6$ ), suggesting that most of the sera contain either antibodies with multiple ganglioside reactivity or multiple antibodies against different gangliosides, a suggestion that was confirmed by twin layer chromatography. The degree of normalised optical density elevation among the patients with the disease was fairly heterogeneous (figs 1 and 2 ). The two patients with CSF oligoclonal bands did not differ in their ganglioside

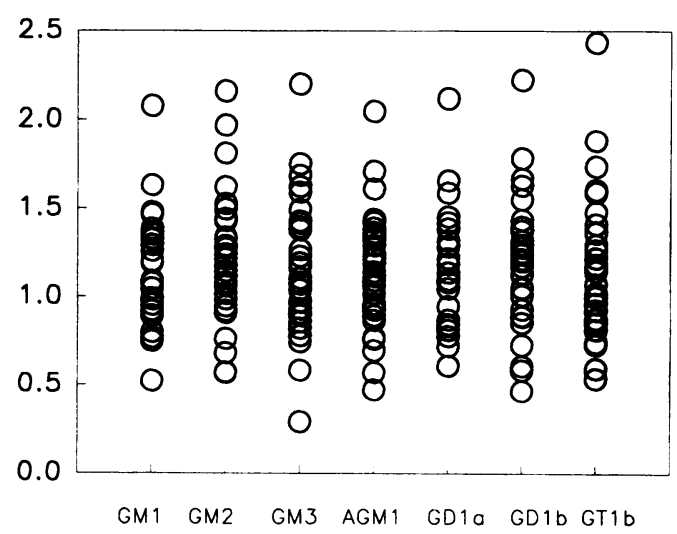


Figure 2 Individual normalised optical density readings for CSF IgG ganglioside antibodies in patients with (left) amyotrophic lateral sclerosis and (right) multiple sclerosis.

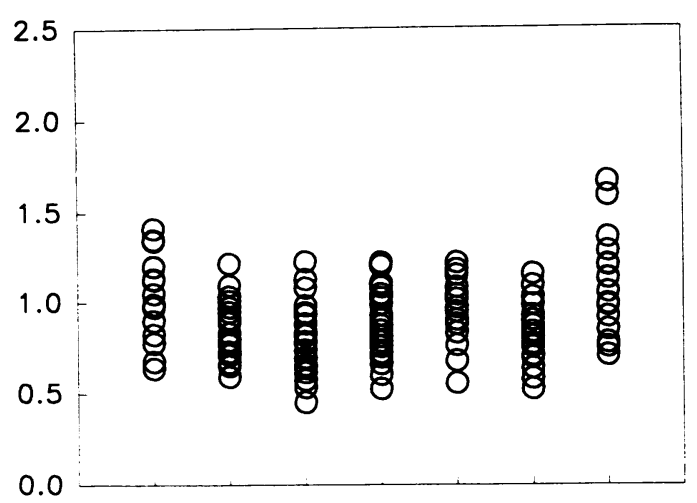

GM1 GM2 GM3 AGM1 GD1a GD1b GT1b

antibody pattern from the other patients with the illness.

A comparison of serum ganglioside antibodies between patients with the disease and controls or those with other neurological disease showed elevated IgG to GM2, GM3, AGM1 and GT1b in the disease but no difference in IgM. Serum reactivity in amyotrophic lateral sclerosis differed from either type of multiple sclerosis only in their higher IgG anti-AGM1 optical density readings.

CSF IgM to all gangliosides except AGM1 was elevated in the patients compared with those with other neurological diseases. CSF IgM to GM2, GM3, GD1a, GD1b, and GT1b was also higher than in chronic progressive multiple sclerosis. On the other hand, patients with chronic progressive multiple sclerosis had higher CSF IgG optical density readings to GM2, GM3, GD1a, and GD1b than patients with amyotrophic lateral sclerosis. CSF IgG antibodies to GM2, GM3, GDla, and GD1b, as well as IgM antibodies to GD1a, GD1b, and GT1b were lower than in relapsing remitting multiple sclerosis. There was no correlation between CSF or serum ganglioside antibodies in amyotrophic lateral or multiple sclerosis.

Discriminant analysis performed on normalised CSF optical density readings to all seven gangliosides allowed correct reclassification of $75 \%$ of the patients with the disease. When variables were selected by stepwise entry depending on Wilk's i reduction, the same classification results were obtained using IgG antibodies to GM2 and GM3, which are low in amyotrophic lateral sclerosis, in association with IgM antibodies to GD1a and GDIb, which are high. None of the controls and $3 \%$ of the patients with multiple sclerosis were falsely classified as having amyotrophic lateral sclerosis.

The same procedure was then applied to serum ganglioside antibodies: this analysis of 35 serum samples from patients with amyotrophic lateral sclerosis, according to their pattern of ganglioside reactivity, allowed correct classification in $83 \%$. Six per cent of the controls with other neurological diseases were mistakenly diagnosed as having the disease (false positives). No single patient with multiple sclerosis received a diagnosis of amyotrophic lateral sclerosis. When only IgG antibodies to GM1, GM2, and AGM1, as

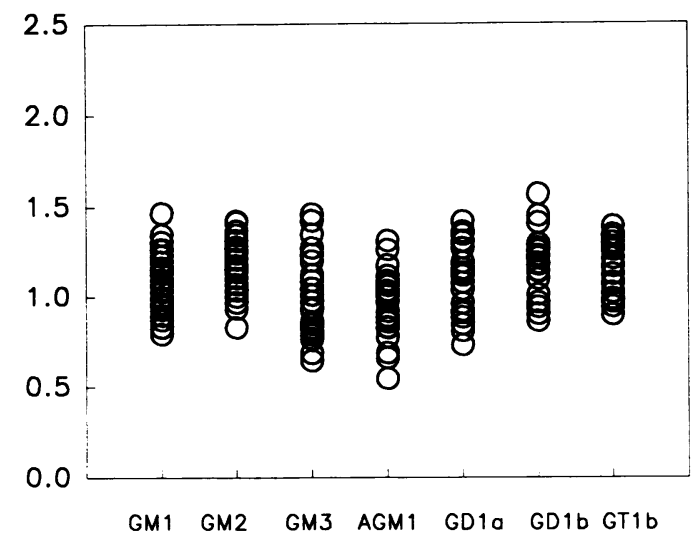

well as IgM antibodies to AGM1 were entered into the analysis, patients with the disease were still correctly identified in $62 \%$, but 14 controls were now misclassified as having the illness. Analysis of variance detected no effect of age, gender or distribution of clinical signs on CSF or serum antibodies in the disease.

\section{Discussion}

The growing number of experimental and clinical studies on altered immune function in amyotrophic lateral sclerosis has not lead to the identification of a consistent abnormality, ${ }^{7}$ except for the few cases associated with plasma cell dyscrasia. ${ }^{214}$ The detection of immune complexes in serum ${ }^{3-5}$ and nervous tissue $^{15}$ as well as of IgG deposits in motor neurons ${ }^{16}$ and lymphocytic infiltrates ${ }^{17}$ and major histocompatibility complex class II antigen expression in nervous tissue ${ }^{18}$ may well be epiphenomenal to neuronal degeneration and does not prove an autoimmune aetiology.

We could not confirm elevated serum IgG or IgM levels or quantitative intrathecal IgG or IgM synthesis or blood-brain barrier breakdown as characteristic features of the disease in our patients. ${ }^{3}$ Previous efforts to identify anti-neuronal protein antibodies in such patients were not successful. ${ }^{19}$ Polyclonal or monoclonal serum antibodies to GM1, which were once thought to be associated with the disease, ${ }^{89}$ have now been recognised to be probably more tightly linked to a lower motor neuron syndrome distinct from amyotrophic lateral sclerosis. ${ }^{12} 20-24$ Moreover, the spectrum of neurological disease with similar levels of serum anti-GM1 antibodies is broad. ${ }^{12} 23$

To our knowledge, the present work is the first comprehensive study of ganglioside antibodies in the CSF of patients with amyotrophic lateral sclerosis. We have identified different patterns of ganglioside antibody reactivity in serum and CSF, both of which are nevertheless characteristic of the disease. This pattern may be the only currently available biochemical test for the diagnosis of amyotrophic lateral sclerosis. The normalised serum optical density readings obtained in this study are lower than those found in patients with multifocal motor neur- 
opathy. ${ }^{1223}$ The lack of correlation between our serum and CSF data suggests that there is a specific compartmental intrathecal immune reaction of $\operatorname{IgM}$ type in the disease. As conventional isoelectric focusing for CSF oligoclonal bands is useful for IgG analysis but does not readily detect $\operatorname{IgM},{ }^{25}$ it is not surprising that oligoclonal bands were seen in only two of our patients. Even sophisticated techniques of IgM oligoclonal band analysis fail to yield positive results in the disease. ${ }^{26} 27$ The lack of elevation of total CSF IgM or the IgM index appears to prove that the immune activation detected in this study cannot be very strong. Nevertheless, we have identified a phenomenon which distinguishes amyotrophic lateral sclerosis from other neurological diseases and normal controls which warrants further investigation. Rather than claiming a primary pathogenetic role of ganglioside antibodies, we suggest that our findings may be related to an alteration of the ganglioside composition in the brain ${ }^{28}$ and spinal cord ${ }^{29}$ of patients with the disease.

1 Matsumoto H, Hanson MR, Chad DA. Amyotrophic lateral sclerosis. Recent advances in pathogenesis and therapeutic trials. Arch Neurol 1988;45:189-202.

2 Younger DS, Rowland LP, Latov N et al. Motor neuron disease and amyotrophic lateral sclerosis: relation of high CSF protein content to paraproteinemia and clinihigh CSF protein content to paraprotein
cal syndromes. Neurology 1990;40:595-9.

3 Apostolski S, Nikolic J, Bugarski-Prokopljevic C, Miletic V, Pavlovic S, Filipovic S. Serum and CSF immunological findings in ALS. Acta Neurol Scand 1991;83:96-8.

4 Oldstone MBA, Wilson CB, Perrin LH, Norris Jr FH. Evidence for immune complex formation in patient with amyotrophic lateral sclerosis. Lancet 1976;2: 169-72.

5 Bartfeld H, Dham C, Donnenfeld $\mathrm{H}$ et al. Immunologica profile of amyotrophic lateral sclerosis patients and their cell-mediated immune response to viral and CNS antigens. Clin Exp Immunol 1982;48:137-47.

6 Appel SH, Stockton-Appel V, Stewart SS, Kerman RH Amyotrophic lateral sclerosis. Associated clinical disorders and immunological evaluations. Arch Neurol 1986; ders and im

7 Drachman DB, Kuncl RW. Amyotrophic lateral sclerosis: an unconventional autoimmune disease? Ann Neurol 1989;26:269-74

8 Pestronk A, Adams RN, Clawson L et al. Serum antibodies to GM1 ganglioside in amyotrophic lateral sclerosis. Neurology 1988;38:1457-61.

$9 \mathrm{Li} \mathrm{F}$, Pestronk A. Autoantibodies to GM1 ganglioside: different reactivity to GM1-liposomes in amyotrophic lateral sclerosis and lower motor neuron disorders. $\mathscr{f}$ Neurol Sci 1991;104:209-14.
10. Weller $M$, Stevens A, Sommer $N$, Wiethölter $H$. Cerebrospinal fluid interleukins, immunoglobulins, and fibronectin in neuroborreliosis. Arch Neurol 1991;48: 837-41.

11 Marcus DM, Latov N, Hsi BP, Gillard BK, and participating laboratories. Measurement and significance of antibodies against GM1 ganglioside. $\mathcal{f}$ Neuroimmunol 1989;25:255-9.

12 Weller M, Stevens A, Sommer N, Dichgans J, Kappler B, Wiethölter H. Ganglioside antibodies. Lack of diagnostic specificity and clinical utility? \& Neurol 1992 (in tic specifi.

13 Stevens A, Weller M, Wiethölter H. Differing CSF and serum ganglioside antibody patterns in chronic progressive and relapsing remitting multiple sclerosis. Neurology 1992;42 (Suppl. 3):247.

14 Shy ME, Rowland LP, Smith T et al. Motor neuron disease and plasma cell dyscrasia. Neurology 1986;36: 1429-36.

15 Donnenfeld H, Kascsak RJ, Bartfeld H. Deposits of IgG and $\mathrm{C} 3$ in the spinal cord and motor cortex of ALS patients. I Neuroimmunol 1984;6:51-7.

16 Engelhardt JI, Appel SH. IgG reactivity in the spinal cord and motor cortex in amyotrophic lateral sclerosis. Arch Neurol 1990;47:1210-6.

17 Troost D, van den Oord JJ, de Jong JMBV, Swaab DF. Lymphocytic infiltration in the spinal cord of patients with amyotroph

18 Lampson LA, Kushner PD, Sobel RA. Strong expression of class II major histocompatibility (MHC) antigens in the absence of detectable $\mathrm{T}$ cell infiltration in amyotrophic lateral sclerosis (ALS) spinal cord. $\mathcal{f}$ Neuropathol Exp Neurol 1988;47:353.

19 Brown RH, Johnson D, Ogonowski M, Weiner HL. Antineural antibodies in the serum of patients with amyotrophic lateral sclerosis. Neurology 1987;37:152-5.

20 Shy ME, Evans VA, Lublin FD et al. Antibodies to GM1 and GDlb in patients with motor neuron disease without plasma cell dyscrasia. Ann Neurol 1989;25:511-3.

21 Salazar-Grueso EF, Routbort MJ, Martin J, Dawson G, Roos RP. Polyclonal IgM anti-GM1 ganglioside antiRoos RP. Polyclonal IgM anti-GM1 ganglioside antibody in patients with motor neu
ants. Ann Neurol 1990;27:558-63.

22 Pestronk A, Chaudhry V, Feldman EL et al. Lower motor neuron syndromes defined by patterns of weakness, nerve conduction abnormalities, and high titres of antiglycolipid antibodies. Ann Neurol 1990;27:316-26.

23 Sadiq SA, Thomas FP, Kilidereas K et al. The spectrum of neurologic disease associated with anti-GM1 antibodies. Neurology 1990;40:1067-72.

24 Lange DJ, Trojaborg W, Latov $\mathrm{N}$ et al. Multifocal motor neuropathy with conduction block: is it a clinical entity? Neurology 1992;42:497-505.

25 Sharief MK, Keir G, Thompson EJ. Glutaraldehydeenhanced immunofixation: a sensitive new method for enhanced immunofixation: a sensitive new method for detecting

26 Sharief MK, Thompson EJ. Immunoglobulin $M$ in the cerebrospinal fluid: an indicator of recent immunological stimulation. $f$ Neurol Neurosurg Psychiatry 1989 52:949-53.

27 Sharief MK, Keir G, Thompson EJ. Intrathecal synthesis of IgM in neurological diseases: a comparison between detection of oligoclonal bands and quantitative estimation. $\mathcal{f}$ Neurol Sci 1990;96:131-42.

28 Rapport MM, Donnenfeld H, Brunner W, Hungund B, Bartfeld H. Ganglioside patterns in amyotrophic lateral sclerosis brain regions. Ann Neurol 1985;18:60-7.

29 Dawson G, Stefansson K. Gangliosides of human spinal cord: aberrant composition of cords from patients with cord: aberrant composition of cords from patients with amyotro $213-20$. 